\title{
n-values of commercial YBCO tapes before and after irradiation by fast neutrons
}

\author{
M.Chudy ${ }^{1,2}$, Z. Zhong ${ }^{2}$, M.Eisterer ${ }^{3}$, T. Coombs ${ }^{2}$ \\ ${ }^{1}$ Graduate School of Technology Management, University of Pretoria, Lynnwood Road, Hatfield, Pretoria 0001, \\ South Africa \\ ${ }^{2}$ Department of Engineering, University of Cambridge, 9 JJ Thomson Avenue, Cambridge CB3 OFA, UK \\ ${ }^{3}$ Vienna University of Technology - Atominstitut, Stadionallee 2, 1020 Vienna, Austria
}

\section{Abstract}

The n-value is an important superconducting parameter, which represents the homogeneity of characterized superconductor as well as thermally activated depinning. In addition n-values are important for the evaluation of pinning mechanisms and pinning forces. n-values are crucial input parameters for the numerical simulations of superconducting tapes, coils and other complicated superconducting applications where E-J power law applies. In this publication, complex measurement data of n-values from different $2^{\text {nd }}$ generation of high temperature superconducting (2G HTS) tapes are presented and analysed. In addition, 2G HTS tapes were step by step irradiated by fast neutron fluences up to $1 \times 10^{22} \mathrm{~m}^{-2}$. n-values of the irradiated tapes, containing additional randomly distributed pinning centres, are presented, analysed and compared with unirradiated samples. Special attention is placed on the underlying physics resulting in power-law part of the I-V curve and on the correlation between critical currents and n-values. The measurements are performed within the temperature range of $50 \mathrm{~K}-85 \mathrm{~K}$ and magnetic fields up to $15 \mathrm{~T}$.

\section{Introduction}

Each I-V curve of a superconducting sample contains a power-law part close to the transition to the dissipative state. This part of the I-V curve can be described by a simple equation:

$$
\mathrm{V} / \mathrm{V}_{\mathrm{c}}=\left(\mathrm{I} / \mathrm{I}_{\mathrm{c}}\right)^{\mathrm{n}}
$$


where $\mathrm{V}$ and $\mathrm{I}$ are the measured voltage and current, $\mathrm{V}_{\mathrm{c}}$ is the voltage criterion, $\mathrm{I}_{\mathrm{c}}$ is the critical current and the n-value the exponent $\mathrm{n}$. Even though the $\mathrm{n}$-value is an important superconducting parameter, results on 2 G HTS tapes are available only in a few recent publications [1-5]. Therefore, a comprehensive overview of experimental n-value data would be beneficial when dealing with numerical modelling of superconductors. In low-temperature superconductors, the exponential relationship (power-law part) in $\mathrm{I}-\mathrm{V}$ is usually explained by the $\mathrm{J}_{\mathrm{c}}$ nonuniformity. Warnes and Larbalestier [6] and Plummer and Evetts [7] successfully developed models based on the $J_{c}$ nonuniformity, but such a mechanism is not so successful in high temperature superconductors. Magnetic flux creep is a mechanism introduced by Anderson and Kim [8,9] and describes a concept of thermally activated flux lines released from pinning centres. This phenomenon is mainly observed at higher temperatures where HTS operate. The rate of this process (R) is exponential, depending on the temperature of the superconductor according to the relation:

$$
R \propto \exp \left(-\frac{U_{e}}{k T}\right)
$$

where $U_{e}$ represents activation energy, $k$ - Boltzmann's constant, $T$ - absolute temperature. This process leads to the redistribution of flux lines resulting in a decrease of the magnetic moment of the superconductor, which is known as magnetic relaxation. Magnetic relaxation in HTS was reviewed by Y. Yeshurun et al. [10], where the direct relation between flux creep and the power law relationship in the I-V curve was pointed out. Finally, studies by J.Z Sun [11] and R. Griessen [12] provided evidence that the power-law relationship is a consequence of the flux creep. However, both effect ,nonuniformity and the flux creep, do not exclude each other and can collaboratively contribute to the exponential part of the I-V curve at the same time. Nowadays, flux creep is considered as the primary reason of the power-law relationship for high temperature superconductors even though some older publications suggested otherwise (i.e. nonunifortmity or inhomogeneity) $[13,14]$. It means that even a "perfectly uniform" HTS would have the power law part of the I-V curve. The n-value of the "perfectly uniform" HTS can be even relatively low in the case of efficient pinning centres with small activation energy. Nonuniformity is not necessarily present in large scale in modern 2G HTS tapes. Generally, the influence of the uniformity factor to the n-value is more important at lower 
temperatures. With increasing temperatures and magnetic fields the uniformity factor becomes less important and importance of flux creep increases. As assumed by the uniformity based models $[13,14]$, critical currents are limited by grain boundaries and not grains. The transition between grain boundary and grain limited currents could be a threshold, where nonuniformity is not the main reason of the power-law relationship. In any case, the n-value always strongly depends on the efficient pinning mechanism in the superconductor.

Measurements of $n$-values after introducing additional pinning centres require special attention. Irradiation by fast neutrons introduces randomly distributed spherical pinning centres with diameters of a few nm [15-20] into a superconductor. It is a proven method of introducing efficient pinning centres into HTS, resulting in critical currents enhancements. Significant critical current enhancement was reported in rather stronger magnetic fields and lower temperatures for several types of tapes [2022].

Regarding the HTS applications, high n-values reduce the losses by operation close to the $\mathrm{J}_{\mathrm{c}}$ and are necessary to operate magnets in the persistence mode. At the same time, high n-values are also associated with unstable behaviour which can cause premature quenching in superconducting machines [23]. Usually, high $\mathrm{n}$-values are considered as an asset of superconductors and rapid n-value reduction at higher magnetic fields can make them unsuitable for any applications. The n-value can vary significantly in different kind of tapes as well as after irradiation. Therefore, measurements were performed on commercial $2 \mathrm{G}$ HTS tapes from three different manufacturers and also after irradiation by fast neutrons.

\section{Samples}

Standard tapes from three different manufacturers were used. The first is the 2G HTS SCS 4045 tape from SuperPower, which is made by Metal Organic Chemical Vapor Deposition (MOCVD) on an Ion Beam Assisted Deposition (IBAD) made MgO template [24]. The YBCO layer is $1 \mu \mathrm{m}$ thick. Two set of samples are characterized, both sets are the SCS 4045 tapes manufactured in 2008 and 2012 respectively. The size of YBCO grains in this kind of tapes is $\sim 1 \mu \mathrm{m}$. The second series of samples are from American Superconductor (AMSC). These tapes have RABiTS ${ }^{\circledR}$ (Rolling Assisted Bi-axially 
Textured Substrates) substrate and Metal Organic Deposited (MOD) YBCO layer ( 1 $\mu \mathrm{m})$. The $4 \mathrm{~mm}$ wide tape is marked as 344 and the 12 mm wide tape as Amperium 8612, with a double HTS Layer [25]. The usual size of YBCO grains in $\operatorname{RABiTS}^{\circledR}$ tapes is 20-50 $\mu \mathrm{m}$ [26]. The third series of samples is coming from Shanghai Superconductor Technology Co.[27]. In these tapes, the MgO template is made by IBAD and Pulsed Laser Deposition (PLD) is used for $1 \mu \mathrm{m}$ thick YBCO layer. The best tested $4 \mathrm{~mm}$ wide sample reached the highest value of critical current. More information about all the samples are listed in Table 1. All the used $4 \mathrm{~mm}$ wide samples were $26 \mathrm{~mm}$ long and the $12 \mathrm{~mm}$ wide samples were $80 \mathrm{~mm}$ long.

\section{Instrumentation}

All the presented instruments are adjusted for transport current characterization of short samples (apx. $3 \mathrm{~cm}$ length) by a standard 4 point method. As data for this study are obtained from several experiments performed by different devices in different laboratories (Low temperature and superconductivity laboratory at Vienna University of Technology, Atominstitut, and EPEC superconductivity laboratory at the University of Cambridge). Most of the samples were characterized by several experimental set-ups. A brief description of experimental instrumentation is given below:

\section{The electromagnet set-ups}

Two electromagnet measurement set-ups were employed for angle-resolved transport measurements, the first one was in Vienna (Atominstitut) and the second one in Cambridge (EPEC superconductivity group). The first set-up consisted of a $1.4 \mathrm{~T}$ water cooled electromagnet and the second of a $800 \mathrm{mT}$ electromagnet where bigger area of homogenous field can be achieved. As for cryostats, a simple tubeshaped vacuum vessel (flask) and a polystyrene box were used. All the measurements were performed in liquid nitrogen. Both set-ups are designed for angle-resolved transport measurements in the maximal Lorentz force configuration. The main difference between the two measurement set-ups is the rotation mechanism. While on the first set-up the holder is statically mounted between the rotating magnet poles, while the holder in the second set-up is rotating and the magnet poles are 
stable. Maximum achievable resolution on both systems is $0.5^{\circ}$. Both sample holders are equipped with Hall sensors and they produce identical results. The Cambridge was used preferred for wider samples reaching very high currents and the irradiated samples were characterized exclusively in Vienna due to safety certificates.

\section{T measurement set-up}

The $6 \mathrm{~T}$ measurement set-up is a helium gas flow cryostat equipped with a $6 \mathrm{~T}$ split coil. The main advantage of this cryostat is a wide temperature range of measurements from about $4.2 \mathrm{~K}$ to $150 \mathrm{~K}$. The horizontal magnetic field allows to perform angle-resolved transport measurements with a rotating sample holder. The rotating sample holder is equipped with a sensitive Hall probe, a Cernox temperature sensor and a fine rotating mechanism with a stepper motor $\left(0.1^{\circ}\right.$ precision $)$. Characterized samples can have lengths up to $30 \mathrm{~mm}$. Indium press contacts are typically used for current and the conductive silver glue is used for the voltage contacts.

\section{T measurement set-up}

The $17 \mathrm{~T}$ measurement set-up is a helium flow cryostat which is equipped with superconducting coils, generating magnetic field up to $17 \mathrm{~T}$ in vertical direction. The Variable Temperature Inset (VTI) has an inner diameter of about $3 \mathrm{~cm}$ and the magnetic field is homogeneous in a vertical length of about $30 \mathrm{~mm}$. These parameters limit the maximum samples lengths to $30 \mathrm{~mm}$. Two sample holders designed for short tapes characterization are available. One places the sample with the $a b$ plane and the other with the $c$-axis parallel to the magnetic field. A 300 A current source was available for the transport characterization.

\section{Triga Mark II reactor}

The TRIGA Mark II was used as an irradiation facility in this work. It is a pool type research reactor that is used for training, research and isotope production (TRIGA - Training, Research, Isotope production, General Atomic) [28]. The reactor has a maximum continuous thermal power of $250 \mathrm{~kW}$, though the power can be increased up to $250 \mathrm{MW}$ for about $40 \mathrm{~ms}$ in the pulse regime. The fuel is in 
the form of an uniform mixture of $8 \mathrm{wt} \%$ uranium, $1 \mathrm{wt} \%$ hydrogen and $91 \mathrm{wt} \%$ zirconium, where the zirconium-hydride is being the main moderator. The maximum neutron flux density of $10^{17} \mathrm{~m}^{-2} \mathrm{~s}^{-1}$ at $250 \mathrm{~kW}$ is reached in the Central Irradiation Facility (CIF). The sample's temperature is estimated to remain below $50{ }^{\circ} \mathrm{C}$ during the irradiation procedure.

\section{Results}

\section{Tapes in low magnetic fields}

All the measurements in this section are performed by the electromagnetic measurement set-ups in liquid nitrogen in fields below or equal to $400 \mathrm{mT}$. As all studied commercial tapes have good grain alignment, the transition from grain boundary limited currents to grain limited currents must occur in this field range, according to [22,29] even well below 400mT. As at low fields the grain boundary limited currents may occur, both nonuniformity and flux creep must be taken into account as the reasons of the exponential part of the $\mathrm{I}-\mathrm{V}$ curve. It is also important to mention that all the measurements are evaluated by a voltage criterion of $1 \mu \mathrm{V} / \mathrm{cm}$ as $U_{c}$ is a locked parameter by fitting with eq.(1). $4 \mathrm{~mm}$ tapes and wider $12 \mathrm{~mm}$ tapes from the three different manufacturers were characterized. In addition, two sets of $4 \mathrm{~mm}$ wide samples from SuperPower were characterized. Critical currents as well as n-values of all types of characterized tapes are listed in table 1. Results from both, newer (2012) and older (2008) tapes are available in figure 1. The results from $4 \mathrm{~mm}$ AMSC and SHSC tapes are shown in figures 2 and 3, respectively. All the angle-resolved measurements were performed in one of the introduced electromagnet measurement set-ups. The results of the n-values are shown together with figures of critical currents for a better illustration of critical current behaviour. Figures with n-values are generally noisier than the figures with critical currents as a consequence of fitting algorithm. n-values and critical currents were calculated from the power law fit of the exponential part of the IV curve. The beginning of the exponential part of the IV curve was determined by first three points above the noise level. The same algorithm was applied for all the measurements from all used measurement set-ups. 
Table 1: Critical currents and n-values of the samples at $77 \mathrm{~K}$ and self-field.

\begin{tabular}{lll}
\hline Sample & Critical current 77 K self-field & n-value \\
\hline SuperPower(2008) $\mathbf{4} \mathbf{~ m m}$ & $98 \mathrm{~A}$ & 28.8 \\
SuperPower(2012) $\mathbf{4 m m}$ & $114 \mathrm{~A}$ & 30.5 \\
SuperPower(2012) $\mathbf{1 2 \mathbf { m m }}$ & $389 \mathrm{~A}$ & 30.1 \\
AMSC 4mm & $92.5 \mathrm{~A}$ & 36 \\
AMSC 12 $\mathbf{m m}$ & $534 \mathrm{~A}$ & 52.2 \\
SHSC 4mm & $167.5 \mathrm{~A}$ & 42.14 \\
\hline
\end{tabular}

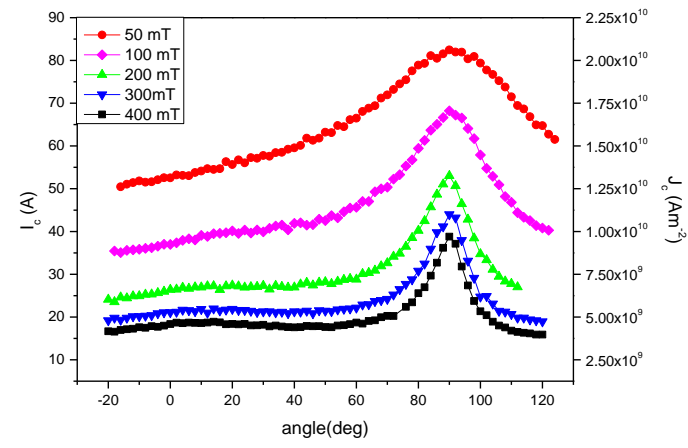

a)

c)

Figure 1: a) b) Critical current and n-values of the SuperPower tape (2008) at $77 \mathrm{~K}$, c) d) critical current and $\mathrm{n}$ values of the SuperPower tape (2012) at $77 \mathrm{~K}$.

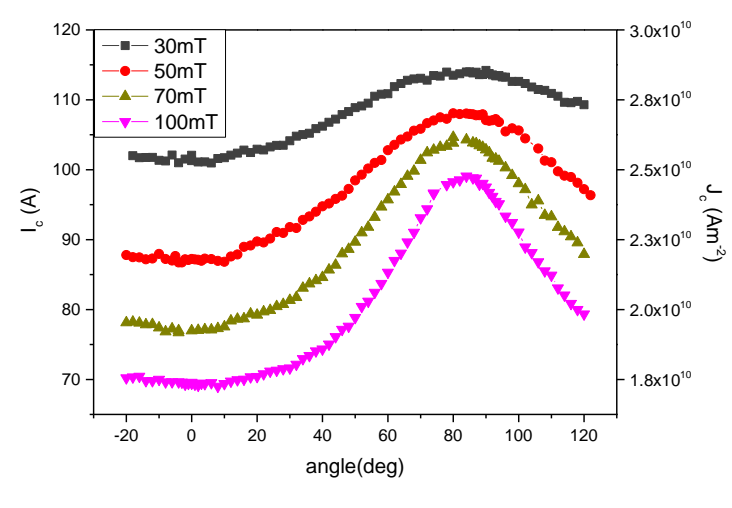

b)
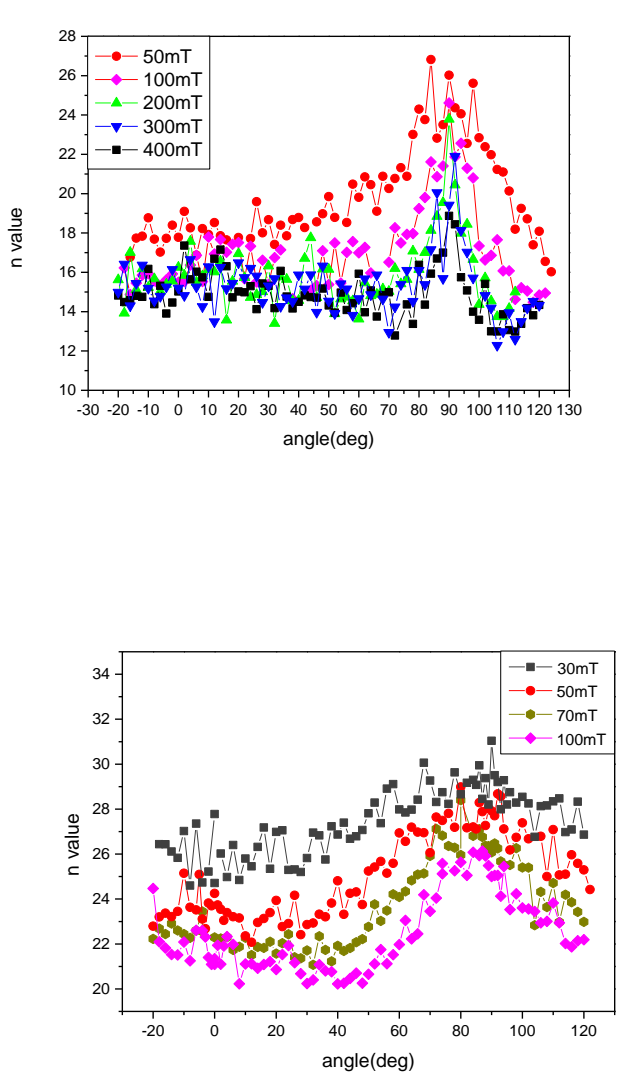

d)

(1)

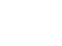



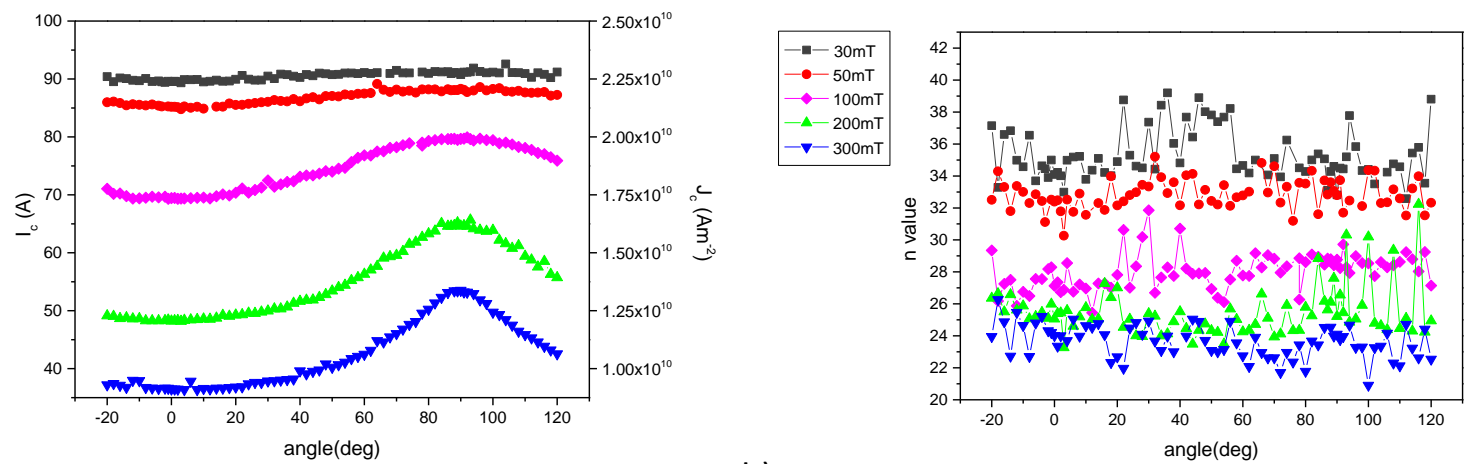

a)

b)

Figure 2: 4mm AMSC tape at 77K: a) critical currents b) n-values

Surprisingly, n-values of the AMSC tape are not dependent on the angle of magnetic field, even though some correlation between n-values and critical current exists also in RABiTS AMSC tape as presented in [2]. Figures 2 and 5 are even showing signs of inverse $J_{c}-n$-value correlation, which will be discussed later in this paper. In numerous experimental studies, it has been observed that n-values are usually correlated with critical currents $[1,2,30]$, which causes that $n$-value varies also with external magnetic field. However, if the correlation is a consequence of relation presented by Zeldov et al. [31], then:

$$
U_{e}=U_{0} \ln \left(\frac{J_{c 0}}{J}\right)
$$

where $\mathrm{U}_{0}$ is the $\mathrm{J}=0$ activation energy (pinning energy), $\mathrm{J}$ is current density and $\mathrm{J}_{\mathrm{c} 0}$ is the critical current density in absence of thermal activation. From (2):

$$
V \propto \frac{d M}{d t} \propto \exp \left(-\frac{U_{e}}{k T}\right)
$$

where $\mathrm{dM} / \mathrm{dT}$ is the rate of magnetization change. Thus, a simple substitution:

$$
\begin{aligned}
& V \propto \frac{J_{c 0}}{J} \exp \left(-\frac{U_{0}}{k T}\right) \propto \exp \left(\ln \left(\frac{J_{c 0}}{J}\right)^{-\frac{U_{0}}{k T}}\right) \propto\left(\frac{J_{c 0}}{J}\right)^{-\frac{U_{0}}{k T}} \propto J^{\frac{U_{0}}{k T}} \\
& n \propto \frac{U_{0}}{k T}, V \propto J^{n} \propto I^{n}
\end{aligned}
$$


It shown that $\mathrm{n}$-value is directly proportional to the pinning energy of flux and not to the $\mathrm{J}_{\mathrm{c}}$. Thus $\mathrm{J}_{\mathrm{c}}=f$ $\left(\mathrm{U}_{0}\right)$, according to (3):

$$
\ln \left(\frac{J_{c 0}}{J_{c}}\right)=\frac{U e c}{U_{0}}
$$

$\mathrm{U}_{\mathrm{ec}}$ is activation energy at the critical current. It can be assumed that $\left(\mathrm{J}_{\mathrm{c} 0}-\mathrm{J}_{\mathrm{c}}\right)$ is higher at higher temperatures and converging to zero at very low temperatures. If $\mathrm{J}_{\mathrm{c}}$ is a function of $(\phi, \mathrm{H}, \mathrm{T})$ according to (7), then $\mathrm{U}_{0}$ is also function of $(\phi, H, T)$, however, the angular dependence of $\mathrm{U}_{0}$ in external magnetic field can be quite different than the one for the $\mathrm{J}_{\mathrm{c}}$. This can be a consequence of flux lines deformation e.g. into staircase like shapes [3,4]. Nonuniformity might play a role in the uncorrelated dependence as well.

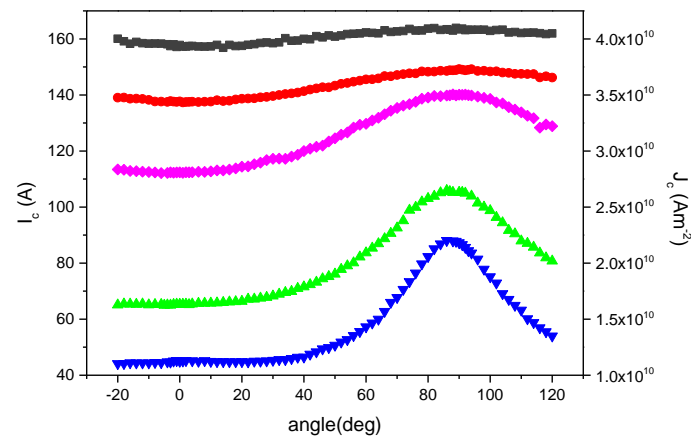

a)

Figure 3: SHSC 4mm wide tape at $77 \mathrm{~K}$ a) critical currents b) n-values

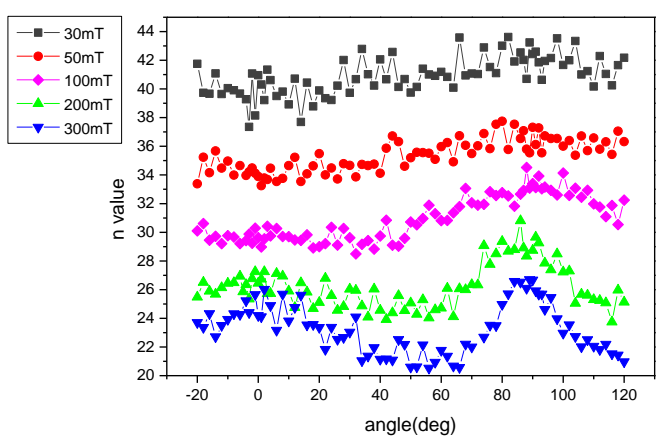

b)

The SHSC sample has shown superior properties. Critical currents were significantly higher than in the other characterized tapes. Although n-values were very high at $30 \mathrm{mT}$, in higher fields they were comparable with tapes from other manufacturers. Anisotropy of the n-values is obvious only from fields above $200 \mathrm{mT}$.

In the next step, characterization of $12 \mathrm{~mm}$ wide tapes from SuperPower(2012) and AMSC was performed (figures 4,5). According to the manufacturers [24, 25], the 12mm tape from SuperPower should have identical structure as the $4 \mathrm{~mm}$ tape. Therefore, approximately three times higher critical currents and similar n-values would be expected compared to the 4mm tape. The AMSC $12 \mathrm{~mm}$ tape contains two HTS layers. The additional layer causes that critical currents should be about 6 times higher than in the case of the $344,4 \mathrm{~mm}$ wide tape. It is relatively difficult to predict the n-values of 
this tape according to the results of the 344 AMSC $4 \mathrm{~mm}$ wide tape. In the case of the SuperPower(2012) tape, $n$-values are slightly higher than for the $4 \mathrm{~mm}$ tape. This difference seems to be insignificant and most likely it is just an effect of different voltage criterion. Wider tape creates more voltage for the same electric field $\mathrm{E}$ and therefore critical electric field $\mathrm{E}_{\mathrm{c}}$, for the wider $12 \mathrm{~mm}$ tape is smaller than $E_{c}$ of $4 \mathrm{~mm}$ tape at the same voltage criterion. For this reason, n-values were calculated for different voltage criteria [32]. The original $1 \mu \mathrm{V} / \mathrm{cm}$ criterion, but also higher $3 \mu \mathrm{V} / \mathrm{cm}$ and $6 \mu \mathrm{V} / \mathrm{cm}$ voltage criteria were used. Although, $\mathrm{n}$-values calculated with higher criterion are slightly lower, no significant difference can be seen between figures $4 \mathrm{~b}$ and $4 \mathrm{c}$. Even though n-values calculated by this enlarged voltage criterion are very similar to the $4 \mathrm{~mm}$ SuperPower(2012) tape, the used enlargement of the voltage criterion is not completely justified due to for example the edge effect of the tapes.

A clear $a b$ peak shift can be observed in figure 4 . This phenomenon was observed in other references [5,33]. It is interesting to note that the shift of the $a b$ peak is obvious also in the case of n-values as well (figure $4 \mathrm{~b}$ ). Change of the criterion seems to be without too much effect by the $12 \mathrm{~mm}$ wide AMSC tape with two YBCO layers. This tape has shown very high n-values for both voltage criteria. The measured n-values are significantly higher especially in low fields if compared to the 344 AMSC $4 \mathrm{~mm}$ wide tape. (figure 5). Most likely, other more complex mechanisms are involved in this phenomenon where identifying of these mechanisms would be a pure speculation at this stage. 


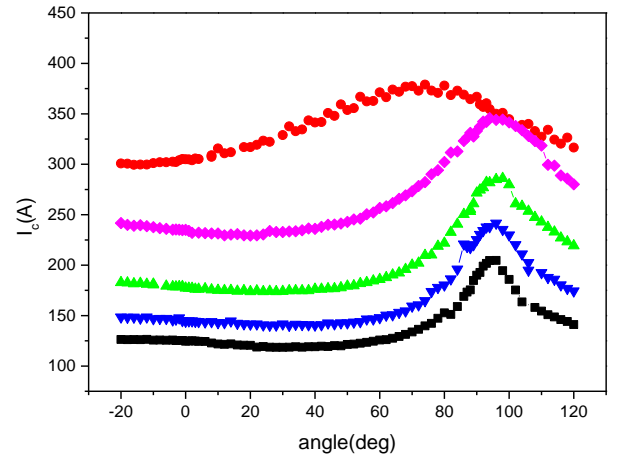

a)

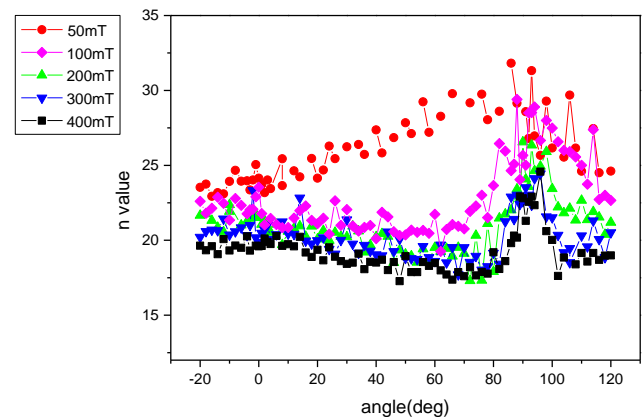

b)

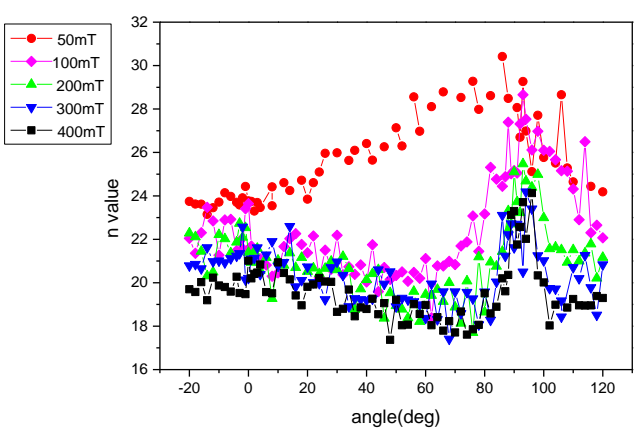

c)

Figure 4: 12mm wide SuperPower(2012) tape a) critical currents b) n-values with criterion $1 \mu \mathrm{V} / \mathrm{cm}$ c) $\mathrm{n}$-values with criterion $3 \mu \mathrm{V} / \mathrm{cm}$ 


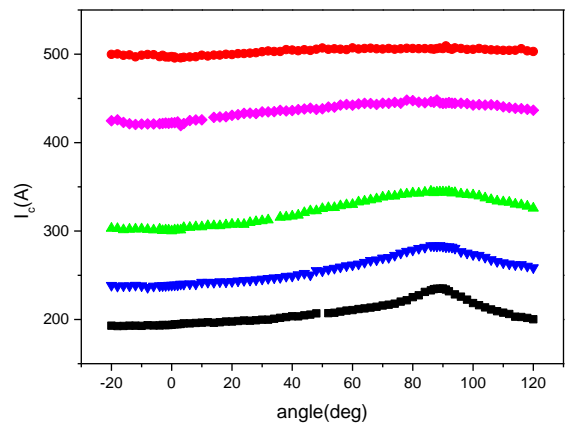

a)

b)
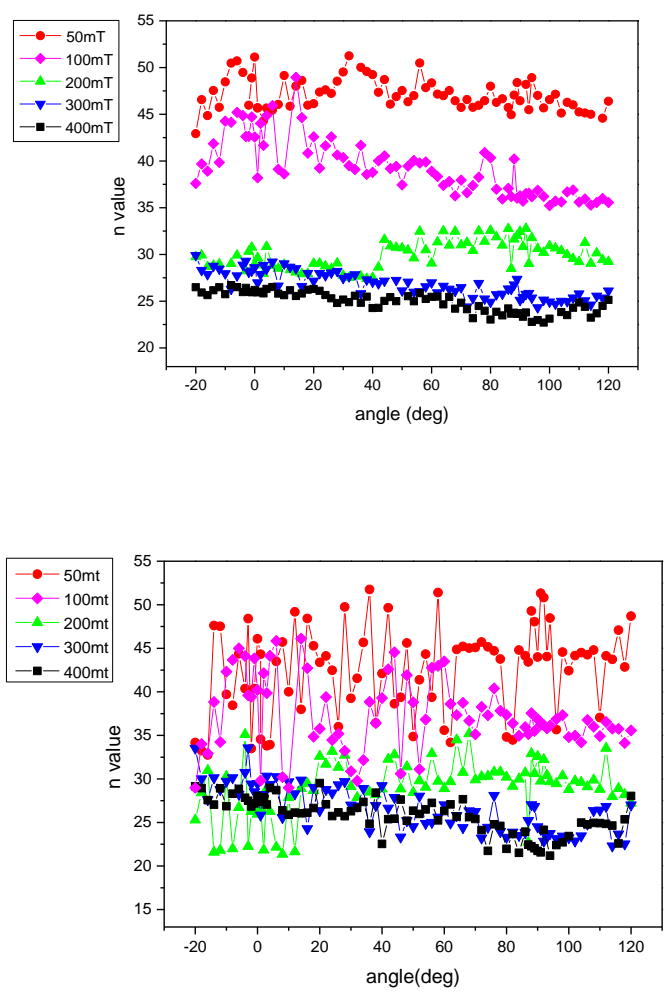

c)

Figure 5:12 mm wide double HTS layer Amperium tape a) critical currents b) n-values with criterion $1 \mu \mathrm{V} / \mathrm{cm} \mathrm{c}) \mathrm{n}$ values with criterion $6 \mu \mathrm{V} / \mathrm{cm}$

\section{Tapes in high magnetic fields and after irradiation}

In this part, results in higher magnetic fields $(B \geq 1 \mathrm{~T})$ are presented. The tapes were irradiated several times by fast neutron fluences and re-measured. The presented graphs (figure 6) consist of results after each irradiation step. The type of artificial defects induced by neutron irradiation strongly depends on the kinetic energy of neutrons. Fast neutrons are neutrons with higher energies $(E \geq 0.1$ $\mathrm{MeV}$ ) and they produce spherical defects of amorphous material with a diameter of a few $\mathrm{nm}$ (so called collision cascades)[15,17-19]. The point defects and clusters of point defects are created by neutrons with lower energies and they can act as effective pinning as well [34]. The Triga Mark reactor II in Vienna (CIF) was used as an irradiation facility in this work. The irradiation levels of fast neutrons applied to the samples were up to a fluences of $1 \times 10^{22} \mathrm{~m}^{-2}$. The other irradiation levels were: $2 \times 10^{21} \mathrm{~m}^{-2}$ and $4 \times 10^{2} \mathrm{~m}^{-2}$. Since the irradiation procedure is a very time consuming process, only the 4 mm wide SuperPower(2008) tapes were characterized. The main time delays are caused by the fact that the samples are becoming radioactive emitters after the neutron irradiation procedure. In order to 
perform the measurements without any health and safety hazards, it is necessary to wait until the radioactivity of the sample is decayed to the acceptable limits.

The measurements were performed in the $6 \mathrm{~T}$ and $17 \mathrm{~T}$ measurement set-ups at various temperatures from $50 \mathrm{~K}$ up to $85 \mathrm{~K}$. Similar studies focused on only critical current enhancement/reduction of $2 \mathrm{G}$ HTS tapes after irradiation are already available [20-22]. In figure 6 are shown the n-value results together with corresponding critical currents.

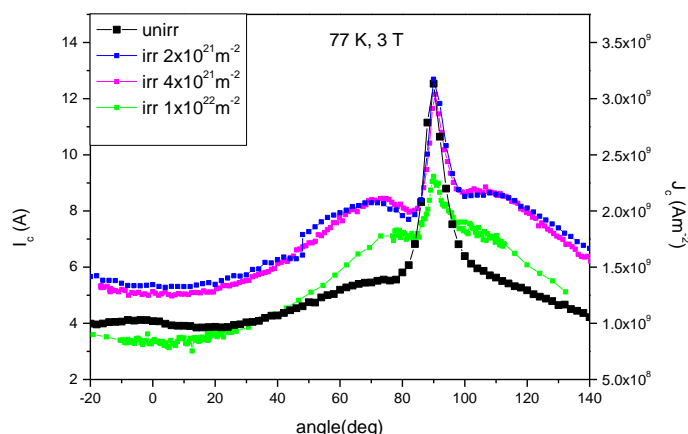

a)

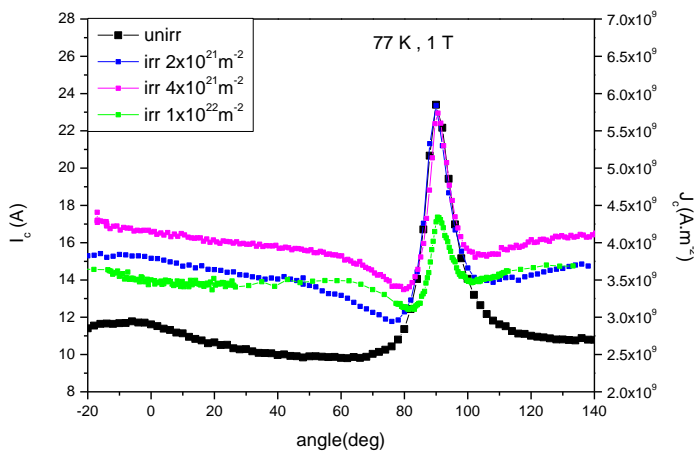

c)

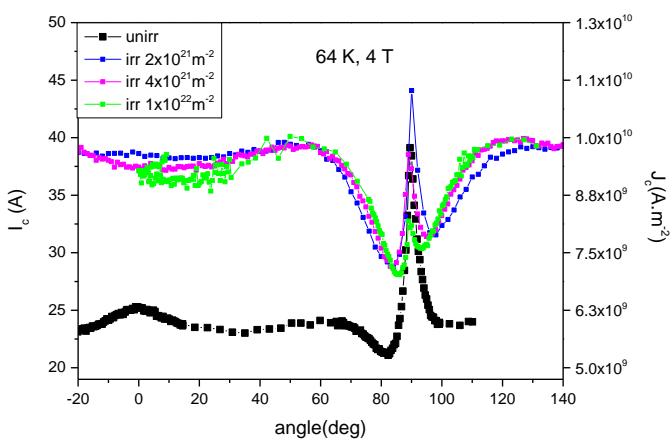

e)

Figure 6: SuperPower(2008) tape characterized after fast neutron irradiation in the $6 \mathrm{~T}$ measurement set-up: a) critical currents $77 \mathrm{~K}, 3 \mathrm{~T}$ b) n-values $77 \mathrm{~K}, 1 \mathrm{~T}$ c) critical currents $77 \mathrm{~K}, 1 \mathrm{~T} \mathrm{~d}$ )n-values $77 \mathrm{~K}$, 1T e) critical currents $64 \mathrm{~K}, 4 \mathrm{~T}$ f) n- values $64 \mathrm{~K}, 4 \mathrm{~T}$.

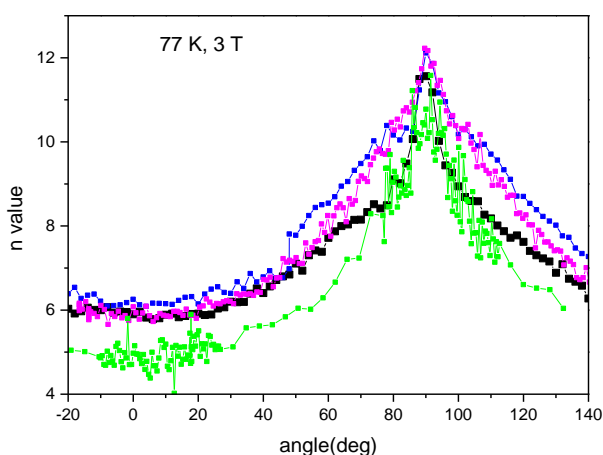

b)

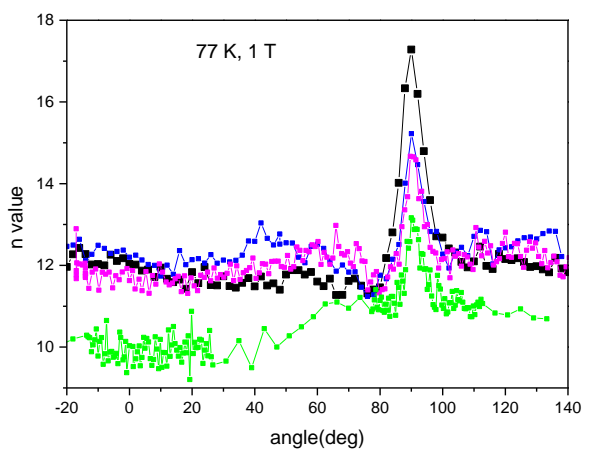

d)

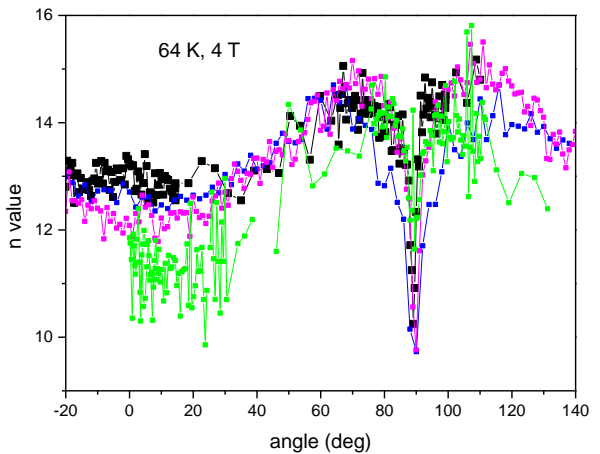

f) 
A significant reduction of $n$-values over the whole scale of measured angles, fields and temperatures is observed at the highest fast neutron fluence $\left(1 \times 10^{22} \mathrm{~m}^{-2}\right)$. At the lower irradiation levels, there are some regions with increase and also with reduction of the n-values. At $64 \mathrm{~K}$, the position of the $\mathrm{J}_{\mathrm{c}}$ peak corresponds to the deep drop of the n-value curves which is in literature known as inverse correlation to the $\mathrm{J}_{\mathrm{c}}$ (figure $6 \mathrm{f}$ ) [3-5]. Despite of this drop, the anisotropy of n-values at $64 \mathrm{~K}$ is rather low. The inverse correlation of the $n$-values by the $J_{c}$ peak has been observed also at $50 \mathrm{~K}$. The inverse $n$-value and $J_{c}$ correlation is commonly related to the staircase flux lines in the superconductor $[3,4]$. Staircase flux lines are usually created in periodic pinning structures of high densities. Intrinsic pinning could be considered as this pinning structure at certain temperatures. Intrinsic pinning is characteristic by the small activation energy of the pinned flux; however, the high density of these pinning centres makes them very efficient at low temperatures when thermal depinning becomes less important. The change of the n-value curve between $77 \mathrm{~K}$ and $64 \mathrm{~K}$ indicates some transition in the role of intrinsic pinning in this range. This transition could cause change of parameters in relation (7), or even transition to a different relation. It was noticed in [31] that the relation between $\mathrm{J}_{\mathrm{c}}$ and $\mathrm{n}$-value can be even linear at certain low current conditions. Nevertheless, it is important to note that all the presented measurements in this section are performed at high fields where critical currents are controlled by grains only and not the grain boundaries as shown in $[22,29]$. This excludes models explaining n-values assuming grain boundary controlled currents and nonuniformity from $[13,14]$. Even the explanation by the staircase flux lines theory is limited to the low fields, as the flux lines they remain mostly straight due to their high numbers in fully penetrated superconductor in higher fields.

After the fast neutron irradiation, randomly distributed efficient pinning centres are introduced into superconducting grains. They are very efficient at wide range orientations of external magnetic fields except orientations where very high density of efficient pinning is present e.g. close to the $a b$ peak. The fast neutron introduced pinning centres can have higher activation energies than original pinning and can enhance n-values. This enhancement is observed in figure 6, especially at $77 \mathrm{~K}$ (figures $6 \mathrm{~b}, \mathrm{~d}$ ). A drop of $n$-values is observed at the fluence of $1 \times 10^{22} \mathrm{~m}^{-2}$. The drop of $\mathrm{n}$-values looks like a 
consequence of very high density pinning centres. All the situations with very high density of efficient pinning centres (high neutron fluences, intrinsic pinning) have always shown smaller n-values. There is probably a threshold value of efficient pinning centres concentration after which the $n$-value starts to be reduced with increasing concentration. This threshold value is obviously strongly dependant on field and temperature. High irradiation fluences cause damage of the crystal structure which can be its reason of loss or partial loss of ability of flux pinning. The consequences are reduced $\mathrm{J}_{\mathrm{c}}$ values close to the $a b$ peak in all the presented measurements (figures 6a,c,e),especially at the highest fluence. The other consequence is probably partial n-value recovery at the $a b$ peak at $64 \mathrm{~K}, 4 \mathrm{~T}$, at the highest neutron fluence, where the flux pinning abilities of the intrinsic pinning centres could be significantly reduced.

None of the mentioned effects is a consequence of nonuniformity in the HTS, which is experimentally proven in the following section of the paragraph. It is well known that neutron irradiation introduces disorder into the YBCO crystals. The commonly observed consequence of this disorder, which is mostly a consequence of already mentioned point defects in the oxygen sublattice, is critical temperature reduction of irradiated samples [15,33]. However if fast neutron irradiation creates nonuniformity in HTS, particular YBCO grains would have different $\mathrm{T}_{\mathrm{c}}$ causing wider transition to the normal state. Therefore, fine $T_{c}$ measurements after each irradiation step were performed. The result (figure 7) shows no influence of fast neutron irradiation on the broadening the transition. It means that the point defects caused by neutron irradiation are homogenously distributed and no additional nonuniformity is created. The homogenous distribution is possible due to their mobility and also relatively high neutron fluences. 


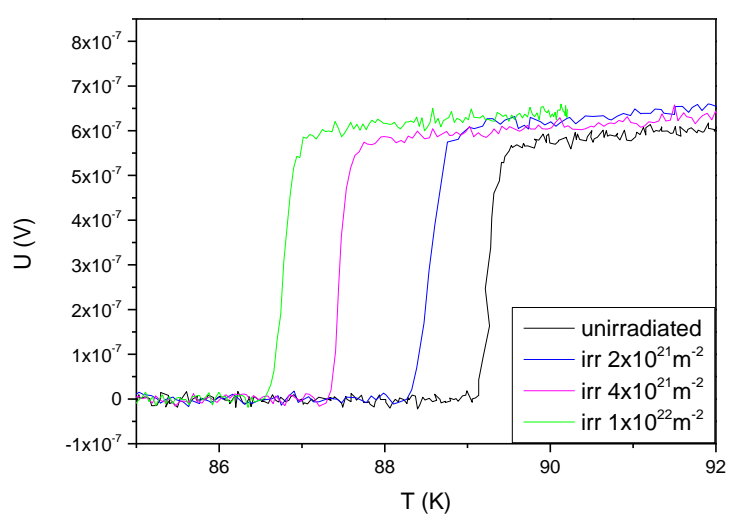

Figure 7: $T_{c}$ transition transport measurements after each irradiation step performed in $17 \mathrm{~T}$ measurement set-up.

The SuperPower(2008) tape was characterized also in high fields up to $15 \mathrm{~T}$ (17 T measurement setup) in two main magnetic field directions. This measurement should confirm results from previous angle-resolved measurements with additional information of n-values in very high fields. Results of these measurements together with measurements after irradiation by fast neutron fluences of $4 \times 10^{21} \mathrm{~m}^{-}$ ${ }^{2}$ are shown in figure 8 . It is worth to note that at lower temperatures such as $64 \mathrm{~K}$ and $50 \mathrm{~K}$, at magnetic fields parallel to the $a b$ planes ( $\mathrm{H} \mathrm{II} \mathrm{ab} \mathrm{-} \mathrm{represents} 90^{\circ}$ in figure 6), n-values are not reduced with increasing magnetic field (after the initial drop at low fields). 


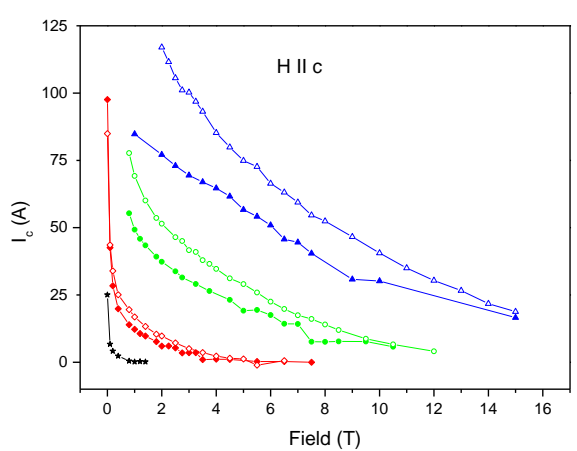

a)

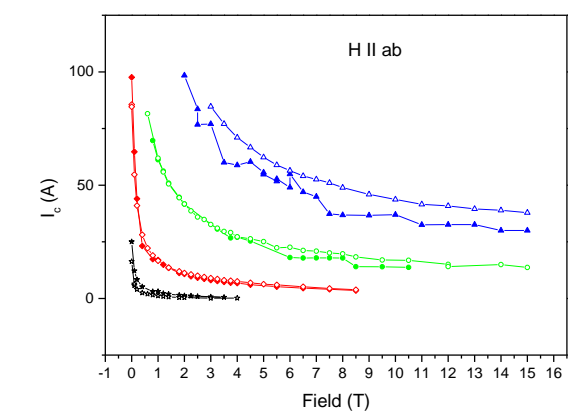

c)

Figure 8: critical currents and n-values at different external magnetic fields, temperatures and irradiation levels: a),b) HIIab, c),d) HIIc.

\section{Summary and conclusions}

n-values of different $4 \mathrm{~mm}$ and $12 \mathrm{~mm}$ wide commercial $2 \mathrm{G}$ HTS tapes at various magnetic fields and temperatures were presented. The performance of the HTS tapes characterized in higher fields is still relatively low. In addition reduction of n-values would enforce them to be operated even at lower currents, which makes them not suitable for most of the possible applications where high fields are required.

Higher n-values and relatively low anisotropy of n-values (compared to $J_{c}$ ) was observed in recent $2 G$ HTS tapes. n-values were studied also after fast neutron irradiation, which introduced randomly distributed spherical defects into the superconductor. Two main factors, nonuniformity and flux creep, are considered as a source the exponential relationship of the I-V curve. Nonuniformity was excluded as the primary factor at higher temperatures and higher fields, where the currents are limited by grains 
and not by the grain boundaries. In addition, it was shown that all the $n$-value changes after fast neutron irradiation cannot be consequence of nonuniformity as irradiation defects were distributed homogenously. Another studied phenomenon was correlation between $n$-values and $\mathrm{J}_{\mathrm{c}}$. It was shown that $n$-value is proportional to pinning energy which usually results into correlation with $\mathrm{J}_{\mathrm{c}}$. However, inverse correlation between $n$-values and $\mathrm{J}_{\mathrm{c}}$ was found under circumstances, where high densities of efficient pinning centres are present.

\section{References}

[1] Jeffrey O. Willis, J. Yates Coulter, and Martin W. Rupich, "n-Value Analysis of PositionDependent Property Variability in Long-Length Coated Conductors", IEEE Trans.Appl. Supercond, Vol. 21, No. 3, 2011.

[2] James Yates Coulter, Terry G. Holesinger, John A. Kennison, Jeffrey O. Willis, Member, IEEE, and Martin W. Rupich, "Nondestructive Investigation of Position Dependent $I_{c}$ Variations in MultiMeter Coated Conductors", IEEE Trans.Appl. Supercond, Vol. 19, No. 3, 2009.

[3] T. Sueyoshi, T. Kotaki, T. Fujiyoshi, F. Mitsugi, T. Ikegami, N. Ishikawa, “Angular dependence of critical current density and n-values in $\mathrm{BaZrO} 3 / \mathrm{YBa} 2 \mathrm{Cu} 3 \mathrm{Oy}$ quasi-multilayered films with columnar defects", Physica C 494, 153-157, 2013.

[4] L. Civale, B. Maiorov, J. L. MacManus-Driscoll, H. Wang, T. G. Holesinger, S. R. Foltyn, A. Serquis, and P. N. Arendt, "Identification of Intrinsic ab-Plane Pinning in YBa2Cu3O7 Thin Films and Coated Conductors", IEEE Trans.Appl. Supercond, Vol. 15, No. 2, 2005.

[5] M. Chudy, M. Eisterer, H.W. Weber, "Asymmetric angular dependence of Jc in coated conductors prior to and after fast neutron irradiation”, Physica C 470, 1300-1303,2010.

[6] W. H. Warnes and D. C. Larbalestier, "Analytical technique for deriving the distribution of critical currents in a superconducting wire,'” Appl. Phys. Lett.48, 1403, 1986.

[7] C. J. G. Plummer and J. E. Evetts, "Dependence of the shape of the resistive transition on composite inhomogeneity in multifilamentary wires," IEEE Trans. Magn.23, 1179, 1987.

[8] P. W. Anderson, “Theory of flux creep in hard superconductors," Phys. Rev. Lett.9, 309, 1962.

[9] P. W. Anderson and Y. B. Kim, "Hard Superconductivity: Theory of the motion of Abrikosov flux lines,' Rev.Mod. Phys.36, 39, 1964.

[10] Y. Yeshurun, A.P. Malozemoff, A. Shaulov, "Magnetic relaxation in high-temperature superconductors", Reviews of Modern Physics, Vol. 68, No. 3, July 1996.

[11] J. Z. Sun, C. B. Eom, B. Lairson, J. C. Bravman, and T. H. Geballe, "Magnetic relaxation, current-voltage characteristics, and possible dissipation mechanisms for high-Tc superconducting thin films of Y-Ba-Cu-O,' Phys. Rev. B 43, 3002, 1991. 
[12] R. Griessen, "Relaxation effects,I-Vcurves and irreversibility lines in high Tc superconductors,"’ Physica C 175,315, 1991.

[13] N. Adamopoulos, J. E. Evetts, , “A model for the V-I characteristics in brick wall-like structures,” IEEE Trans.Appl. Supercond. 3, 1257, 1993.

[14] E.Evetts, B.A.Glowacki, P.L.Sampson, M.G.Blamire, N.McN.Alford, M.A.Harmer, "Relation of the n-value of the resistive transition to microstructure and inhomogeneity for YBCO wires" IEEE Trans. on Magnetics, Vol. 25, No. 2, 1989.

[15]F. M. Sauerzopf, H. P. Wiesinger, H. W. Weber, G. W.Crabtree, "Analysis of pinning effects in $\mathrm{YBa}_{2} \mathrm{Cu}_{3} \mathrm{O}_{7-\delta}$ single crystals after fast neutron irradiation" Phys. Rev. B, vol. 51,No.9 p. 6002, 1995.

[16] M. Okada and T. Kawakubo, Radiation Effects and Defects in Solids, vol. 108, no. 2-4, pp. 137144, 1989.

[17] M C Frischherz, M A Kirk, J P Zhang and Weber H W, "Transmission electron microscopy of defect cascades in $\mathrm{YBa}_{2} \mathrm{Cu}_{3} \mathrm{O}_{7-\delta}$ produced by ion irradiation”, 1993 Phil. Mag. A 671347.

[18]M.C. Frischherz, M.A. Kirk , J. Farmer , L.R. Greenwood , H.W. Weber ," Defect cascades produced by neutron irradiation in $\mathrm{YBa}_{2} \mathrm{Cu}_{3} \mathrm{O}_{7-\delta}$ " Physica C 232, 309-327, 1994.

[19] M. C. Frischherz, M. A. Kirkl, j. Farmer, and H. W. Weber, "Defect Cascades Produced by Neutron Irradiation in in $\mathrm{YBa} 2 \mathrm{Cu} 307$. Superconductors, 7th International Workshop on Critical Currents in Superconductors", Alpbach, Tyrol/Austria, January 24-27, 1994.

[20] M. Chudy, R. Fuger, M. Eisterer, and H. W. Weber, „Characterization of Commercial YBCO Coated Conductors After Neutron Irradiation” IEEE Trans.Appl. Supercond, Vol. 21, No. 3, 2011.

[21] R. Fuger, M. Eisterer and H. W. Weber, "YBCO coated conductors for fusion magnets", IEEE Trans.Appl. Supercond, Vol. 19, No. 3, 2009.

[22] M. Eisterer, R. Fuger, M. Chudy, F. Hengstberger and H. W.Weber, Neutron irradiation of coated conductors, Supercond. Sci. Technol. 23, 014009 (6pp), 2010.

[23] Arup K. Ghosh," V-I transition and n-value of multifilamentary LTS and HTS wires and cables", ICMC'03 Topical Conference, Enschede, The Netherlands, May 25-28,2003.

[24] http://www.superpower-inc.com

[25] http://www.amsc.com

[26] M. Weigand, S. C. Speller, G. M. Hughes, N. A. Rutter, S. Lozano-Perez, C. R. M. Grovenor, J. H. Durrell, "Individual grain boundary properties and overall performance of metal-organic deposition coated conductors”, Phys. Rev. B 81, 174537, 2010.

[27] http://www.shsctec.com/en/index.asp

[28] Weber H W, Bock H, Unfried E and Greenwood L R, "Neutron dosimetry and damage calculations for the TRIGA MARK-II reactor in Vienna” J. Nucl. Mater. 137, 236-40, 1986.

[29] S. Tonies, A. Vostner, and H. W. Weber, "Determination of inter- and intragranular currents in high temperature superconducting tapes and coated conductors", J. Appl. Phys, 92, 5, 2628,2002. 
[30] Zhang Hong, Wu Hong, Ren Cong-Xin and Chen Guo-Liang, "The anisotropy of the currentcarrying characteristics of YBCO film fabricated by magnetron sputtering", Supercond. Sci. Technol. 7, 354363, 1994.

[31] Zedlov E, Amer N M,Krren G,Gupta A,Gambino R J and McElfresh M W, "Optical and electrical enhancement of flux creep in YBa2Cu3O7- $\delta$ epitaxial films”, Phys. Rev. Lett.62, $3093,1989$.

[32] A. V. Pan, I. A. Golovchanskiy and S. A. Fedoseev, "Critical current density: Measurements vs. reality", EPL, 103, 17006, 2013.

[33] Z. Chen, F. Kametani, Y. Chen, Y. Xie, V. Selvamanickam and D. C. Larbalestier," A high critical current density MOCVD coated conductor with strong vortex pinning centers suitable for very high field use" Supercond. Sci. Technol. 22 (2009) 055013 (5pp), 2009.

[34] M. Chudy, M. Eisterer, H W Weber, J. Veternikova, S. Sojak, V. Slugen,” Point defects in YBa2Cu3O7x studied using positron annihilation" Supercond. Sci. Technol. 25 (2012) 075017 (5pp),2012. 